\title{
Multiplicity of solutions for a singular $p$-laplacian elliptic equation
}

\author{
by Wen-Shu Zhou and XiaO-DAN Wei (Dalian)
}

\begin{abstract}
The existence of two continuous solutions for a nonlinear singular elliptic equation with natural growth in the gradient is proved for the Dirichlet problem in the unit ball centered at the origin. The first continuous solution is positive and maximal; it is obtained via the regularization method. The second continuous solution is zero at the origin, and follows by considering the corresponding radial ODE and by sub-sup solutions method.
\end{abstract}

1. Introduction. Consider the nonlinear problem

$$
\left\{\begin{array}{l}
-\operatorname{div}\left(|\nabla u|^{p-2} \nabla u\right)=f(x)-\frac{\lambda}{u^{m}}|\nabla u|^{p}, \quad u>0, \quad \text { in } \Omega, \\
u=0 \text { on } \partial \Omega .
\end{array}\right.
$$

Here $\Omega$ is a bounded domain in $\mathbb{R}^{N}(N \geq 2)$ with smooth boundary $\partial \Omega$, $1<p<\infty, \lambda \neq 0, m \in \mathbb{R}$, and $f(x)$ is measurable in $\Omega$. Such problems arise in the theory of non-Newtonian fluids (see [AM], [MP, [MRS]).

For $m>0$, the equation in (1.1) is singular at points where $u=0$.

When the lower order term $B(x, u, \eta)$ has no singularity at $u=0$, the following nonlinear elliptic problems have been studied extensively (see [A], [AAA], [AB], [ADP], [BBM], [BMP1], [BMP2], [BO], [BST], [CC], [DGP], $[\mathrm{DB}], \mathrm{DN}],[\mathrm{FPR},[\mathrm{G}],[\mathrm{GT}],[\mathrm{L},[\mathrm{LU}], \mathrm{OP},[\mathrm{P},[\mathrm{PS},[\mathrm{T}],[\mathrm{Tr}, \mathrm{YC}]$ and references therein): Find $u \in W^{1, p}(\Omega) \cap L^{\infty}(\Omega)$, such that

$$
\left\{\begin{array}{l}
-\operatorname{div} A(x, u, \nabla u)+B(x, u, \nabla u)=0 \quad \text { in } \mathcal{D}^{\prime}(\Omega), \\
u-h \in W_{0}^{1, p}(\Omega),
\end{array}\right.
$$

where $h \in W^{1, p}(\Omega) \cap L^{\infty}(\Omega),-\operatorname{div} A$ is a Leray-Lions operator from $W_{0}^{1, p}(\Omega)$ into $W^{-1, p^{\prime}}(\Omega)\left(p>1, p^{\prime}>1,1 / p+1 / p^{\prime}=1\right)$, which includes the $p$ laplacian, and $B$ is a nonlinear lower order term with natural growth in

2010 Mathematics Subject Classification: Primary 35J25; Secondary 35J75.

Key words and phrases: p-laplacian equation, singularity, natural growth, multiplicity. 
the gradient. Under some additional assumptions on $A$ and $B$, the existence and regularity of solutions were studied in a large number of papers: see for instance Abdellaoui, Dall'Aglio and Peral [ADP, Amann [A], Bensoussan, Boccardo and Murat [BBM], Boccardo, Murat and Puel [BMP1, BMP2], Cho and Choe [CC], Dall'Aglio, Giachetti and Puel [DGP], Drábek and Nicolosi [DN], Ferone, Posteraro and Rakotoson [FPR, Grenon and Trombetti [GT], Orsina and Puel [OP] and Porretta and Segura de León [PS] for the existence and DiBenedetto [DB], Ladyzhenskaya and Ural'tseva [LU], Lieberman $[\mathrm{L}$ and Tolksdorf $[\mathrm{T}]$ for the regularity.

When the lower order term $B$ may be singular at $u=0$, to the best of our knowledge, the problem has received little attention. In [MRS], Michaux, Rakotoson and Shen studied a class of quasilinear mixed equations of LerayLions type; however, they only considered the case where the boundary function has a positive lower bound and established the existence and local regularity of solutions with the same lower bound, whereas the positive lower bound means disappearance of singularity of the lower order term and plays a dominant role in their proof. It is of interest to consider the singularity of the lower order term and to study the existence of solutions in $W_{0}^{1, p}(\Omega) \cap L^{\infty}(\Omega)$.

In the present paper, we investigate the existence and multiplicity of solutions of problem (1.1) with $m>1$ and $\lambda>0$; we extend the existence results of [AMA, $\mathrm{PV}, \overline{\mathrm{Z} 1}]$ and the multiplicity result of [Z2].

In [Z1], Zhou considered a class of nonlinear singular elliptic problems including (1.1) with $m=1$, and established the existence of a positive solution under the assumptions $\lambda \geq(p-1) / p$ and $f \in L^{\infty}(\Omega)$ with $\operatorname{ess} \inf _{\Omega} f>0$. In the case of $p=2$, problem [1.1) was studied in [AMA, [PV] and [Z2]. For $0<m \leq 1$, Arcoya and Martínez-Aparicio AMA proved the existence of a positive distributional solution under the assumptions $\lambda>0$ and $f \in L^{\infty}(\Omega)$ with $\inf _{\Omega^{\prime}} f>0$ for any $\Omega^{\prime} \subset \subset \Omega$. For $m=1$, Porru and Vitolo [PV] established the existence of a positive classical solution via a substitution if $\lambda>0$ and $f \equiv$ const $>0$. For $4 / 3>m>1$, Zhou [Z2] obtained two different continuous solutions: one that is maximal and positive and another that is zero at the origin, if $\Omega=B_{1}:=\left\{x \in \mathbb{R}^{N} ;|x|<1\right\}, f(x)=\bar{f}(|x|)$, $\bar{f} \in C[0,1]$ with $\bar{f}>0$ on $[0,1]$ and $\lambda>\inf _{r \geq 1} \mathcal{H}(r)$, where

$$
\mathcal{H}(r)=\frac{2(m-1)+N(2-m)}{2} r^{m-1}+\frac{(2-m)^{2} \max _{[0,1]} \bar{f}}{4} r^{m-2} .
$$

The second solution can be obtained by considering the corresponding ODE with mixed boundary conditions and by the sub-sup solutions method.

We point out that in the case of $m=1$, by a certain transformation (see [Z1]), problem (1.1) can be transformed into either a Dirichlet problem or a boundary blowup problem without a gradient term; such problems have 
been studied extensively in the past years (see the references cited in [Z1]). It is worth noting that for $m>1$, it seems to be impossible to transform the equation in (1.1) into an equation without a gradient term.

As seen in Section 2, the case $m \geq 1$ is essentially different from the case $0<m<1$. Indeed, we show that in the latter case, problem (1.1) admits at most one solution (see Corollary 2.5), while in the former case it has at least two solutions. The main idea of dealing with the multiplicity is similar to that in [Z2]. Roughly speaking, the proof consists of two steps. In the first step, we obtain a maximal, positive, continuous solution for a general domain $\Omega$ (see Theorem 2.6), for which our idea is based on the regularization method, monotonicity technique and Lemma 3.7. In the second step, we will assume $\Omega=B_{1}$ and $f(x)=\bar{f}(|x|)$ and consider the radial ODE corresponding to the equation in (1.1):

$$
\left(\left|u^{\prime}\right|^{p-2} u^{\prime}\right)^{\prime}+\frac{N-1}{r}\left|u^{\prime}\right|^{p-2} u^{\prime}-\frac{\lambda}{u^{m}}\left|u^{\prime}\right|^{p}+\bar{f}(r)=0, \quad 0<r<1 .
$$

To get the second solution, we consider 1.2 with the boundary conditions

$$
u(1)=u(0)=u^{\prime}(0)=0 .
$$

A function $u \in C^{1}[0,1]$ is called a solution to problem $(1.2)$ and 1.3 if $u>0$ in $(0,1),\left|u^{\prime}\right|^{p-2} u^{\prime} \in C^{1}(0,1)$, and it satisfies (1.2) and (1.3). By the sub-sup solutions method, we prove that under some additional assumptions on $m, \lambda$ and $\bar{f}$, problem (1.2) and (1.3) has a positive solution $u$ (see the proof of Theorem 2.7). Let $w(x)=u(r)$ with $r=|x|$. Then $w(x)$ is a solution with $w(0)=0$ if $\Omega=B_{1}$ and some assumptions on $m, \lambda$ and $\bar{f}$ are satisfied (see Theorem 2.7). Thus problem (1.1) may have at least two solutions (see Theorem 2.8).

This paper is organized as follows. In Section 2, we state the main results. The other sections are devoted to the proofs of these results.

2. Main results. Denote

$$
\begin{aligned}
& \mathcal{F}=W^{1, p}(\Omega) \cap L^{\infty}(\Omega), \quad \mathcal{F}_{0}=W_{0}^{1, p}(\Omega) \cap L^{\infty}(\Omega), \\
& \mathcal{F}_{0}^{+}=\left\{w \in \mathcal{F}_{0} ; w \geq 0 \text { a.e. in } \Omega\right\} .
\end{aligned}
$$

Definition 2.1. A function $u \in \mathcal{F}$ is called a sup-solution of the equation in (1.1) if $u>0$ a.e. in $\Omega,|\nabla u|^{p} / u^{m} \in L^{1}(\Omega)$, and

$$
\int_{\Omega}\left(|\nabla u|^{p-2} \nabla u \nabla \varphi+\frac{\lambda}{u^{m}}|\nabla u|^{p} \varphi-f(x) \varphi\right) d x \geq 0, \quad \forall \varphi \in \mathcal{F}_{0}^{+} .
$$

Similarly, a function $u \in \mathcal{F}$ is called a sub-solution of the equation in $(1.1)$ if $u>0$ a.e. in $\Omega,|\nabla u|^{p} / u^{m} \in L^{1}(\Omega)$, and it satisfies the converse inequality. 
Definition 2.2. A function $u \in \mathcal{F}_{0}$ is called a solution of problem 1.1 if $u>0$ a.e. in $\Omega,|\nabla u|^{p} / u^{m} \in L^{1}(\Omega)$, and

$$
\int_{\Omega}\left(|\nabla u|^{p-2} \nabla u \nabla \varphi+\frac{\lambda}{u^{m}}|\nabla u|^{p} \varphi-f(x) \varphi\right) d x=0, \quad \forall \varphi \in \mathcal{F}_{0} .
$$

Proposition 2.3. Let $\lambda>0$ and $f \in L^{1}(\Omega)$ with $f \leq 0$ a.e. in $\Omega$. Then problem (1.1) has no solution.

Proof. Assume that $u$ is a solution of problem 1.1. Then $u \in \mathcal{F}_{0}^{+}$. Substituting $\varphi=u$ into the integral equality in Definition 2.2 yields $\int_{\Omega}|\nabla u|^{p} d x$ $=0$, which implies that $u=0$ a.e. in $\Omega$, a contradiction.

Proposition 2.4. Let $\lambda \geq 0$ and $m>0$ be constants, and let $f \in L^{q}(\Omega)$ with $f(x) \geq 0$ a.e. in $\Omega$, where $1 / p+1 / q=1$. Assume that $u_{2}$ and $u_{1}$ are a sup-solution and a sub-solution of the equation in (1.1), respectively, with $u_{2} \geq u_{1}$ on $\partial \Omega$.

(i) If $0<m<1$, then $u_{2} \geq u_{1}$ a.e. in $\Omega$.

(ii) If $m \geq 1$, and if there exist positive constants $c_{2}, c_{1}$ such that $u_{i} \geq$ $c_{i}(i=1,2)$, then $u_{2} \geq u_{1}$ a.e. in $\Omega$.

Here the precise meaning of " $u_{2} \geq u_{1}$ on $\partial \Omega$ " is that " $\left(u_{1}-u_{2}\right)_{+} \in$ $W_{0}^{1, p}(\Omega)$ ", where $s_{+}=\max \{0, s\}$.

Proof. Let $\xi=\lambda /(p-1), \mu_{m}=0$ if $0<m<1$ and $\mu_{m}=\min \left\{c_{2}, c_{1}\right\}$ if $m \geq 1$, and define $g:\left[\mu_{m}, \infty\right) \rightarrow \mathbb{R}$ by

$$
\begin{aligned}
& g(s)=\int_{\mu_{m}}^{s} e^{\frac{\xi}{m-1} y^{1-m}} d y \quad \text { if } m \neq 1, \\
& g(s)= \begin{cases}\frac{s^{1-\xi}}{1-\xi} & (\xi \neq 1) \\
\ln (s) & (\xi=1)\end{cases}
\end{aligned}
$$

Clearly, $g^{\prime}(s)>0$ and $g^{\prime \prime}(s) \leq 0$, for all $s>\mu_{m}$. Then it follows from Definition 2.1 that

$$
\begin{aligned}
& \int_{\Omega}\left(\left|\nabla g\left(u_{2}\right)\right|^{p-2} \nabla g\left(u_{2}\right) \nabla \varphi-\left(g^{\prime}\left(u_{2}\right)\right)^{p-1} f(x) \varphi\right) d x \geq 0, \\
& \int_{\Omega}\left(\left|\nabla g\left(u_{1}\right)\right|^{p-2} \nabla g\left(u_{1}\right) \nabla \varphi-\left(g^{\prime}\left(u_{1}\right)\right)^{p-1} f(x) \varphi\right) d x \leq 0
\end{aligned}
$$

for any $\varphi \in \mathcal{F}_{0}^{+}$, so

$$
\begin{aligned}
\int_{\Omega}\left(\left|\nabla g\left(u_{1}\right)\right|^{p-2} \nabla g\left(u_{1}\right)-\right. & \left.\left|\nabla g\left(u_{2}\right)\right|^{p-2} \nabla g\left(u_{2}\right)\right) \nabla \varphi d x \\
+ & \int_{\Omega} f(x)\left(\left(g^{\prime}\left(u_{2}\right)\right)^{p-1}-\left(g^{\prime}\left(u_{1}\right)\right)^{p-1}\right) \varphi d x \leq 0 .
\end{aligned}
$$


Note that $\varphi=\left(g\left(u_{1}\right)-g\left(u_{2}\right)\right)_{+} \in \mathcal{F}_{0}^{+}$. Substituting it into the above integral inequality yields

$$
\begin{array}{r}
\int_{\Omega}\left(\left|\nabla g\left(u_{1}\right)\right|^{p-2} \nabla g\left(u_{1}\right)-\left|\nabla g\left(u_{2}\right)\right|^{p-2} \nabla g\left(u_{2}\right)\right) \nabla\left(g\left(u_{1}\right)-g\left(u_{2}\right)\right)_{+} d x \\
+\int_{\Omega} f(x)\left(\left(g^{\prime}\left(u_{2}\right)\right)^{p-1}-\left(g^{\prime}\left(u_{1}\right)\right)^{p-1}\right)\left(g\left(u_{1}\right)-g\left(u_{2}\right)\right)_{+} d t \leq 0 .
\end{array}
$$

Since $g^{\prime}>0$ and $g^{\prime \prime} \leq 0$ in $\left(\mu_{m}, \infty\right)$, we obtain

$$
f(x)\left(\left(g^{\prime}\left(u_{2}\right)\right)^{p-1}-\left(g^{\prime}\left(u_{1}\right)\right)^{p-1}\right)\left(g\left(u_{1}\right)-g\left(u_{2}\right)\right)_{+} \geq 0 \text { a.e. in } \Omega,
$$

and hence

$$
\int_{\Omega}\left(\left|\nabla g\left(u_{1}\right)\right|^{p-2} \nabla g\left(u_{1}\right)-\left|\nabla g\left(u_{2}\right)\right|^{p-2} \nabla g\left(u_{2}\right)\right) \nabla\left(g\left(u_{1}\right)-g\left(u_{2}\right)\right)_{+} d x \leq 0 .
$$

By the inequality (cf. [D])

$$
\left(|\eta|^{p-2} \eta-\left|\eta^{\prime}\right|^{p-2} \eta^{\prime}\right) \cdot\left(\eta-\eta^{\prime}\right) \geq C\left(|\eta|+\left|\eta^{\prime}\right|\right)^{p-2}\left|\eta-\eta^{\prime}\right|^{2}
$$

for any $\eta, \eta^{\prime} \in \mathbb{R}^{N}$, where $C$ is a positive constant depending only on $p$, one arrives at

$$
\int_{\Omega}\left(\left|\nabla g\left(u_{1}\right)\right|+\left|\nabla g\left(u_{2}\right)\right|\right)^{p-2}\left|\nabla\left(g\left(u_{1}\right)-g\left(u_{2}\right)\right)_{+}\right|^{2} d x=0 .
$$

This shows that $\left(g\left(u_{1}\right)-g\left(u_{2}\right)\right)_{+}=0$ a.e. in $\Omega$, which implies that $u_{2} \geq u_{1}$ a.e. in $\Omega$.

As an immediate consequence of Proposition 2.4, we obtain

Corollary 2.5. Let $\lambda>0$ and $0<m<1$ be constants, and let $f \in$ $L^{q}(\Omega)$ with $f \geq 0$ a.e. in $\Omega$, where $1 / p+1 / q=1$. Then problem (1.1) admits at most one solution in $\mathcal{F}_{0}^{+}$.

The present paper will focus on the case of $m>1$. We first obtain the existence of a solution for the general domain $\Omega$.

TheOREM 2.6. Let $p>1, \lambda>0,2 p /(p+1)>m>1$, and assume $f \in L^{\infty}(\Omega)$ with $\operatorname{ess}_{\inf } \Omega f(x)>0$. Then problem (1.1) admits one maximal, positive solution $u$ in $C_{\mathrm{loc}}^{\alpha}(\Omega) \cap C(\bar{\Omega})$ for some $\alpha \in(0,1)$.

For $\Omega=B_{1} \equiv\left\{x \in \mathbb{R}^{N} ;|x|<1\right\}$, we establish the existence of the second solution of problem (1.1), which can be stated as follows.

Theorem 2.7. Let $\Omega=B_{1}$ and $f(x) \equiv \bar{f}(|x|)$, and let $p>m>1$. Assume that $\bar{f} \in C[0,1]$ with $\bar{f}>0$ on $[0,1]$. If $\lambda>\inf _{r \geq 1} \mathcal{X}(r)$, then problem (1.1) admits one solution $w$ in $C^{1}(\bar{\Omega})$ with $w>0$ in $\Omega \backslash\{0\}$ and $w(0)=0$. Moreover, there exist two positive constants $C_{2}$ and $C_{1}$ with $C_{2} \geq C_{1}$ such that

$$
C_{1} \leq|x|^{(m-p) / p} w(x) \leq C_{2}, \quad 0 \leq|x| \leq 1 / 4 .
$$


Here $\mathcal{X}: \mathbb{R}^{+} \rightarrow \mathbb{R}^{+}$is defined by

$$
\mathcal{X}(r)=\frac{p(m-1)+N(p-m)}{p} r^{m-1}+\frac{(p-m)^{p} \max _{[0,1]} \bar{f}}{p^{p}} r^{m-p} .
$$

By Theorems 2.6-2.7, we immediately obtain the following multiplicity result.

THEOREM 2.8. Let $\Omega=B_{1}$ and $f(x) \equiv \bar{f}(|x|)$, and let $p>1,2 p /(p+1)$ $>m>1$. Assume that $\bar{f} \in C[0,1]$ with $\bar{f}>0$ on $[0,1]$. If $\lambda>\inf _{r \geq 1} \mathcal{X}(r)$, then problem (1.1) has at least two solutions.

REMARK 2.9. Let $p>m>1$, and denote $S^{*}=\left\{\begin{array}{rl}S_{0}, & S_{0} \geq 1, \\ 1, & S_{0}<1,\end{array} \quad S_{0}=\left(\frac{(p-m)^{p+1} \max _{[0,1]} \bar{f}}{p^{p-1}(m-1)[p(m-1)+N(p-m)]}\right)^{1 /(p-1)}\right.$. Then $\inf _{s \geq 1} \mathcal{X}(s)=\mathcal{X}\left(S^{*}\right)$. Indeed, since $\lim _{s \rightarrow 0^{+}} \mathcal{X}(s)=\lim _{s \rightarrow \infty} \mathcal{X}(s)=$ $\infty, \mathcal{X}(s)$ must reach a minimum at some $s \in(0, \infty)$ satisfying $\mathcal{X}^{\prime}(s)=0$, which gives $s=S_{0}$, so $\inf _{s>0} \mathcal{X}(s)=\mathcal{X}\left(S_{0}\right)$. Since $\mathcal{X}^{\prime}(s) \geq 0$ for all $s \geq S_{0}$, we see that $\inf _{s \geq 1} \mathcal{X}(s)=\mathcal{X}\left(S_{0}\right)$ if $S_{0} \geq 1$, and $\inf _{s \geq 1} \mathcal{X}(s)=\mathcal{X}(1)$ if $S_{0}<1$.

3. Proof of Theorem 2.6. Let $\epsilon \in(0,1 / 2)$, and define $H_{\epsilon}: \Omega \times \mathbb{R} \times$ $\mathbb{R}^{N} \rightarrow \mathbb{R}$ by

$$
H_{\epsilon}(x, s, \xi)=\lambda \frac{\operatorname{sgn}(s)}{[I(s)+\epsilon]^{m}}|\xi|^{p}-f(x),
$$

where $I(s)=s$ if $s \geq 0, I(s)=0$ if $s<0$. Clearly, we have

$$
\left|H_{\epsilon}(x, s, \xi)\right| \leq \frac{\lambda}{\epsilon^{m}}|\xi|^{p}+|f|_{\infty}
$$

for almost every $(x, s, \xi) \in \Omega \times \mathbb{R} \times \mathbb{R}^{N}$. Hence it follows from Theorem 1 in [BMP1] that for any fixed $\epsilon \in(0,1 / 2)$, there exists a function $u_{\epsilon} \in \mathcal{F}_{0}$ such that

$$
\int_{\Omega}\left(\left|\nabla u_{\epsilon}\right|^{p-2} \nabla u_{\epsilon} \nabla \varphi+H_{\epsilon}\left(x, u_{\epsilon}, \nabla u_{\epsilon}\right) \varphi\right) d x=0, \quad \forall \varphi \in \mathcal{F}_{0} .
$$

Lemma 3.1. $u_{\epsilon} \geq 0$ a.e. in $\Omega$ for all $\epsilon \in(0,1 / 2)$.

Proof. Since $u_{\epsilon} \in \mathcal{F}_{0}$, we have $\left(u_{\epsilon}\right)_{-} \in \mathcal{F}_{0}$, where $s_{-}=\max \{0,-s\}$. Substituting $\varphi=\left(u_{\epsilon}\right)_{-}$into (3.1) yields

$$
\int_{\Omega}\left(\left|\nabla u_{\epsilon}\right|^{p-2} \nabla u_{\epsilon} \nabla\left(u_{\epsilon}\right)_{-}+\lambda \frac{\operatorname{sgn}\left(u_{\epsilon}\right)\left(u_{\epsilon}\right)_{-}}{\left[I\left(u_{\epsilon}\right)+\epsilon\right]^{m}}\left|\nabla u_{\epsilon}\right|^{p}-f(x)\left(u_{\epsilon}\right)_{-}\right) d x=0 .
$$

This leads to

$$
\int_{\Omega}\left(\left|\nabla\left(u_{\epsilon}\right)_{-}\right|^{p}+\lambda \frac{\left|\left(u_{\epsilon}\right)_{-}\right|}{\left[I\left(u_{\epsilon}\right)+\epsilon\right]^{m}}\left|\nabla u_{\epsilon}\right|^{p}\right) d x=-\int_{\Omega} f(x)\left(u_{\epsilon}\right)_{-} d x \leq 0,
$$


which implies that

$$
\int_{\Omega}\left|\nabla\left(u_{\epsilon}\right)_{-}\right|^{p} d x=0
$$

therefore, $\left(u_{\epsilon}\right)_{-}=0$ a.e. in $\Omega$, i.e. $u_{\epsilon} \geq 0$ a.e. in $\Omega$.

By Lemma 3.1, one derives from (3.1) that

$$
\int_{\Omega}\left(\left|\nabla u_{\epsilon}\right|^{p-2} \nabla u_{\epsilon} \nabla \varphi+\frac{\lambda}{\left(u_{\epsilon}+\epsilon\right)^{m}}\left|\nabla u_{\epsilon}\right|^{p} \varphi-f(x) \varphi\right) d x=0, \quad \forall \varphi \in \mathcal{F}_{0} .
$$

Denote $U_{\epsilon}=u_{\epsilon}+\epsilon$. Then $U_{\epsilon} \geq \epsilon$, and

$$
\int_{\Omega}\left(\left|\nabla U_{\epsilon}\right|^{p-2} \nabla U_{\epsilon} \nabla \varphi+\frac{\lambda}{U_{\epsilon}^{m}}\left|\nabla U_{\epsilon}\right|^{p} \varphi-f(x) \varphi\right) d x=0, \quad \forall \varphi \in \mathcal{F}_{0} .
$$

Next our aim is to show that the $\operatorname{limit}_{\lim _{\epsilon \rightarrow 0^{+}}} u_{\epsilon}(x)=u(x)$ exists for almost every $x \in \Omega$, and the limit function $u$ is a solution of problem (1.1). Before giving the proof, we emphasize that the main difficulty is twofold: singularity and natural growth of the nonlinear lower order term. To overcome the difficulties, we will establish a locally uniform positive lower bound of $u_{\epsilon}$ and prove the strong compactness:

$$
\nabla U_{\epsilon}^{(p-m) / p} \rightarrow \nabla u^{(p-m) / p} \quad \text { strongly in } L^{1}(\Omega)\left(\epsilon \rightarrow 0^{+}\right) .
$$

Due to the singularity, it seems difficult to deal with (3.4) as in the papers mentioned above. Our method relies on the monotonicity of $u_{\epsilon}$ in $\epsilon$, Lemma 3.7 and a compactness argument.

It is worth noting that, to establish (3.4), the condition $\lambda \geq(p-1) / p$ is needed in [Z1] for $m=1$, while for $m>1$ the present paper does not impose any condition on $\lambda$ except $\lambda>0$ (see the proof of Lemma 3.6). Moreover, we have to require $m<2 p /(p+1)$ in order to apply Lebesgue's dominated convergence theorem to the proof of (3.4). To show (3.4), we need to establish some nontrivial, uniform estimates on $u_{\epsilon}$. The following lemma gives uniform upper and lower bounds for $u_{\epsilon}$.

Lemma 3.2. For all $\epsilon \in(0,1 / 2)$,

$$
\Phi \geq u_{\epsilon} \geq C \Phi^{p /(p-m)} \quad \text { a.e. in } \Omega,
$$

where $C>0$ is independent of $\epsilon$, and $\Phi \in C_{0}^{1}(\bar{\Omega})$ denotes the unique positive solution of the problem

$$
-\operatorname{div}\left(|\nabla v|^{p-2} \nabla v\right)=|f|_{\infty} \quad \text { in } \mathcal{D}^{\prime}(\Omega), \quad v \in C_{0}^{1}(\bar{\Omega}),
$$

(see [BMP1] for the existence of $\Phi$ in $W_{0}^{1, p}(\Omega) \cap L^{\infty}(\Omega)$, [L] for the global $C^{1, \alpha}$ regularity and $[\mathrm{D}$ for the positivity). 
Proof. As $\lambda>0$, it follows from (3.2) that

$$
\int_{\Omega}\left(\left|\nabla u_{\epsilon}\right|^{p-2} \nabla u_{\epsilon} \nabla \varphi-|f|_{\infty} \varphi\right) d x \leq 0, \quad \forall \varphi \in \mathcal{F}_{0}^{+},
$$

thus, by Proposition 2.4, one obtains $u_{\epsilon} \leq \Phi$ a.e. in $\Omega$.

Below we show the second estimate in (3.5). Let $W_{\epsilon}=V+\epsilon$ with $V=$ $C \Phi^{p /(p-m)}$, where $C \in(0,1)$ satisfies

$$
\left(\frac{C p}{p-m}\right)^{p-1}|f|_{\infty} \max _{\bar{\Omega}} \Phi^{m(p-1) /(p-m)}+\frac{\lambda C^{p-m} p^{p}}{(p-m)^{p}} \max _{\bar{\Omega}}|\nabla \Phi|^{p} \leq \underset{\Omega}{\operatorname{essinf}} f(x) .
$$

Clearly, $W_{\epsilon} \in W^{1, p}(\Omega) \cap L^{\infty}(\Omega)$. By simple calculations and using the properties of $\Phi$, we deduce that

$$
\begin{aligned}
& -\operatorname{div}\left(\left|\nabla W_{\epsilon}\right|^{p-2} \nabla W_{\epsilon}\right)+\lambda \frac{\left|\nabla W_{\epsilon}\right|^{p}}{W_{\epsilon}^{m}}-f(x) \\
& \leq-\operatorname{div}\left(|\nabla V|^{p-2} \nabla V\right)+\lambda \frac{|\nabla V|^{p}}{V^{m}}-\underset{\Omega}{\operatorname{essinf}} f(x) \\
& =-\left(\frac{C p}{p-m}\right)^{p-1} \Phi^{m(p-1) /(p-m)} \operatorname{div}\left(|\nabla \Phi|^{p-2} \nabla \Phi\right) \\
& -\frac{m(p-1)(C p)^{p-1}}{(p-m)^{p}} \Phi^{p(m-1) /(p-m)}|\nabla \Phi|^{p}+\frac{\lambda C^{p-m} p^{p}}{(p-m)^{p}}|\nabla \Phi|^{p}-\underset{\Omega}{\operatorname{essinf}} f(x) \\
& =\left(\frac{C p}{p-m}\right)^{p-1}|f|_{\infty} \Phi^{m(p-1) /(p-m)}-\frac{m(p-1)(C p)^{p-1}}{(p-m)^{p}} \Phi^{p(m-1) /(p-m)}|\nabla \Phi|^{p} \\
& +\frac{\lambda C^{p-m} p^{p}}{(p-m)^{p}}|\nabla \Phi|^{p}-\underset{\Omega}{\operatorname{essinf}} f(x) \\
& \leq\left(\frac{C p}{p-m}\right)^{p-1}|f|_{\infty} \max _{\bar{\Omega}} \Phi^{m(p-1) /(p-m)}+\frac{\lambda C^{p-m} p^{p}}{(p-m)^{p}} \max _{\bar{\Omega}}|\nabla \Phi|^{p}-\underset{\Omega}{\operatorname{essinf}} f(x) \\
& \leq 0 \quad \text { in } \mathcal{D}^{\prime}(\Omega) \text {. }
\end{aligned}
$$

By Proposition 2.4 and (3.3), we derive that $U_{\epsilon} \geq W_{\epsilon}$ a.e. in $\Omega$, i.e. $u_{\epsilon} \geq V$ a.e. in $\Omega$.

By (3.5), it is easy to derive that for any compact subset $\Omega^{\prime}$ of $\Omega$, there exists a positive constant $C_{0}$, independent of $\epsilon$, such that

$$
u_{\epsilon} \geq C_{0} \quad \text { a.e. in } \Omega^{\prime} \text {, }
$$

therefore

$$
\left|H_{\epsilon}\left(x, u_{\epsilon}(x), \xi\right)\right| \leq \frac{\lambda}{C_{0}^{m}}|\xi|^{p}+|f|_{\infty}
$$

for almost every $x \in \Omega^{\prime}$ and for all $\xi \in \mathbb{R}^{N}$. Consequently, all assumptions of Theorem 6.1 in MRS (see also $[\mathrm{R},[\mathrm{RT}$ ) for local Hölder continuity are satisfied. We conclude that $u_{\epsilon}$ is uniformly bounded in $C^{\alpha}\left(\Omega^{\prime}\right)$ for some 
$\alpha \in(0,1)$. Using the Arzelà-Ascoli theorem, there exist a subsequence of $\left\{u_{\epsilon}\right\}$, still denoted by $\left\{u_{\epsilon}\right\}$, and a function $u \in C_{\text {loc }}^{\alpha}(\Omega)$, such that, as $\epsilon \rightarrow 0^{+}$,

$$
u_{\epsilon} \rightarrow u \text { uniformly in } \Omega^{\prime} \text {. }
$$

Hence, $u$ is continuous in $\Omega$ and satisfies, by (3.5),

$$
\Phi \geq u \geq C \Phi^{p /(p-m)} \quad \text { in } \Omega .
$$

Then $\lim _{x \rightarrow \partial \Omega} u(x)=0$. Define $u=0$ on $\partial \Omega$. Then $u \in C(\bar{\Omega})$.

The following lemma shows the monotonicity of $u_{\epsilon}$ in $\epsilon$.

LemmA 3.3. $u_{\epsilon_{2}} \geq u_{\epsilon_{1}}$ in $\Omega$ for $1 / 2>\epsilon_{2}>\epsilon_{1}>0$.

Proof. Let $V_{\epsilon_{2}}=u_{\epsilon_{2}}+\epsilon_{1}$. Then $U_{\epsilon_{1}}-V_{\epsilon_{2}} \in \mathcal{F}_{0}$. It follows from the equality (3.3) with $\epsilon=\epsilon_{2}$ that

$$
\int_{\Omega}\left(\left|\nabla V_{\epsilon_{2}}\right|^{p-2} \nabla V_{\epsilon_{2}} \nabla \varphi+\frac{\lambda}{V_{\epsilon_{2}}^{m}}\left|\nabla V_{\epsilon_{2}}\right|^{p} \varphi-f(x) \varphi\right) d x \geq 0, \quad \forall \varphi \in \mathcal{F}_{0}^{+} .
$$

By Proposition 2.4, we see that $V_{\epsilon_{2}} \geq U_{\epsilon_{1}}$ in $\Omega$, that is, $u_{\epsilon_{2}} \geq u_{\epsilon_{1}}$ in $\Omega$.

From Lemma 3.3 and (3.6), we derive that

$$
\begin{gathered}
u_{\epsilon} \geq u \quad \text { in } \Omega, \\
u_{\epsilon} \rightarrow u \quad \text { in } \Omega\left(\epsilon \rightarrow 0^{+}\right) .
\end{gathered}
$$

Lemma 3.4. $\nabla u_{\epsilon} \rightarrow \nabla u$ strongly in $L^{p}(\Omega)\left(\epsilon \rightarrow 0^{+}\right)$.

Proof. Substituting $\varphi=u_{\epsilon}$ into $(3.2)$ yields

$$
\int_{\Omega}\left|\nabla u_{\epsilon}\right|^{p} d x+\lambda \int_{\Omega} \frac{u_{\epsilon}}{\left(u_{\epsilon}+\epsilon\right)^{m}}\left|\nabla u_{\epsilon}\right|^{p} d x=\int_{\Omega} f(x) u_{\epsilon} d x,
$$

therefore, using the first estimate of (3.5) yields

$$
\int_{\Omega}\left|\nabla u_{\epsilon}\right|^{p} d x \leq C,
$$

from which and (3.9) we deduce that $\nabla u \in L^{p}(\Omega)$, and up to a subsequence

$$
\nabla u_{\epsilon} \rightarrow \nabla u \quad \text { weakly in } L^{p}(\Omega)\left(\epsilon \rightarrow 0^{+}\right) .
$$

Then substituting $\varphi=u_{\epsilon}-u$ into 3.2 yields

$$
\begin{aligned}
\int_{\Omega}\left|\nabla u_{\epsilon}\right|^{p-2} \nabla u_{\epsilon}\left(\nabla u_{\epsilon}-\right. & \nabla u) d x \\
& +\lambda \int_{\Omega} \frac{u_{\epsilon}-u}{\left(u_{\epsilon}+\epsilon\right)^{m}}\left|\nabla u_{\epsilon}\right|^{p} d x=\int_{\Omega} f(x)\left(u_{\epsilon}-u\right) d x .
\end{aligned}
$$

By (3.8), we have

$$
\int_{\Omega}\left|\nabla u_{\epsilon}\right|^{p-2} \nabla u_{\epsilon}\left(\nabla u_{\epsilon}-\nabla u\right) d x \leq \int_{\Omega} f(x)\left(u_{\epsilon}-u\right) d x,
$$


therefore

$$
\varlimsup_{\epsilon \rightarrow 0^{+}} \int_{\Omega}\left|\nabla u_{\epsilon}\right|^{p-2} \nabla u_{\epsilon}\left(\nabla u_{\epsilon}-\nabla u\right) d x \leq 0 .
$$

Noticing

$$
\lim _{\epsilon \rightarrow 0^{+}} \int_{\Omega}|\nabla u|^{p-2} \nabla u\left(\nabla u_{\epsilon}-\nabla u\right) d x=0,
$$

we obtain

$$
\varlimsup_{\epsilon \rightarrow 0^{+}} \int_{\Omega}\left(\left|\nabla u_{\epsilon}\right|^{p-2} \nabla u_{\epsilon}-|\nabla u|^{p-2} \nabla u\right)\left(\nabla u_{\epsilon}-\nabla u\right) d x \leq 0 .
$$

Recalling the inequality (2.1), we find that

$$
\lim _{\epsilon \rightarrow 0^{+}} \int_{\Omega}\left(\left|\nabla u_{\epsilon}\right|^{p-2} \nabla u_{\epsilon}-|\nabla u|^{p-2} \nabla u\right)\left(\nabla u_{\epsilon}-\nabla u\right) d x=0,
$$

so

$$
\int_{\Omega}\left(\left|\nabla u_{\epsilon}\right|+|\nabla u|\right)^{p-2}\left|\nabla u_{\epsilon}-\nabla u\right|^{2} d x \rightarrow 0 \quad\left(\epsilon \rightarrow 0^{+}\right) .
$$

From this and using Hölder's inequality we get

$$
\begin{aligned}
\int_{\Omega} \mid \nabla u_{\epsilon}- & \left.\nabla u\right|^{p} d x \\
& \leq \int_{\Omega}\left(\left|\nabla u_{\epsilon}\right|+|\nabla u|\right)^{p-1}\left|\nabla u_{\epsilon}-\nabla u\right| d x \\
& =\int_{\Omega}\left(\left|\nabla u_{\epsilon}\right|+|\nabla u|\right)^{p / 2} \frac{\left|\nabla u_{\epsilon}-\nabla u\right|}{\left(\left|\nabla u_{\epsilon}\right|+|\nabla u|\right)^{1-p / 2}} d x \\
& \leq\left(\int_{\Omega}\left(\left|\nabla u_{\epsilon}\right|+|\nabla u|\right)^{p} d x\right)^{1 / 2} \cdot\left(\int_{\Omega} \frac{\left|\nabla u_{\epsilon}-\nabla u\right|^{2}}{\left(\left|\nabla u_{\epsilon}\right|+|\nabla u|\right)^{2-p}} d x\right)^{1 / 2} \\
& \leq C\left(\int_{\Omega} \frac{\left|\nabla u_{\epsilon}-\nabla u\right|^{2}}{\left(\left|\nabla u_{\epsilon}\right|+|\nabla u|\right)^{2-p}} d x\right)^{1 / 2} \rightarrow 0 \quad\left(\epsilon \rightarrow 0^{+}\right) .
\end{aligned}
$$

Lemma 3.4 immediately implies that

$$
\nabla u_{\epsilon} \rightarrow \nabla u \quad \text { a.e. in } \Omega\left(\epsilon \rightarrow 0^{+}\right) .
$$

Lemma 3.5. For all $\epsilon \in(0,1 / 2)$, we have

$$
\int_{\Omega} \frac{\left|\nabla u_{\epsilon}\right|^{p}}{\left(u_{\epsilon}+\epsilon\right)^{m}} d x \leq \frac{1}{\lambda} \int_{\Omega} f(x) d x
$$

i.e.

$$
\int_{\Omega}\left|\nabla U_{\epsilon}^{(p-m) / p}\right|^{p} d x \leq \frac{(p-m)^{p}}{\lambda p^{p}} \int_{\Omega} f(x) d x .
$$


Proof. Clearly, $\frac{u_{\epsilon}}{u_{\epsilon}+\sigma} \in \mathcal{F}_{0}$ for any $\sigma>0$. Substituting it into 3.2 yields $\sigma \int_{\Omega} \frac{\left|\nabla u_{\epsilon}\right|^{p}}{\left(u_{\epsilon}+\sigma\right)^{2}} d x+\lambda \int_{\Omega} \frac{\left|\nabla u_{\epsilon}\right|^{p}}{\left(u_{\epsilon}+\epsilon\right)^{m}} \frac{u_{\epsilon}}{u_{\epsilon}+\sigma} d x=\int_{\Omega} f(x) \frac{u_{\epsilon}}{u_{\epsilon}+\sigma} d x$,

therefore

$$
\lambda \int_{\Omega} \frac{\left|\nabla u_{\epsilon}\right|^{p}}{\left(u_{\epsilon}+\epsilon\right)^{m}} \frac{u_{\epsilon}}{u_{\epsilon}+\sigma} d x \leq \int_{\Omega} f(x) \frac{u_{\epsilon}}{u_{\epsilon}+\sigma} d x .
$$

Note that $u_{\epsilon}>0$ in $\Omega$. Then for any fixed $\epsilon \in(0,1 / 2)$ we have

$$
\frac{u_{\epsilon}}{u_{\epsilon}+\sigma} \rightarrow 1 \quad \text { in } \Omega\left(\sigma \rightarrow 0^{+}\right) \text {. }
$$

Letting $\sigma \rightarrow 0^{+}$and using Lebesgue's dominated convergence theorem, we obtain

$$
\lambda \int_{\Omega} \frac{\left|\nabla u_{\epsilon}\right|^{p}}{\left(u_{\epsilon}+\epsilon\right)^{m}} d x \leq \int_{\Omega} f(x) d x .
$$

Now passing to the limit in $(3.13)$ as $\epsilon \rightarrow 0^{+}$and using Fatou's lemma and noticing (3.9), (3.7) and (3.12) yield

$$
\int_{\Omega} \frac{|\nabla u|^{p}}{u^{m}} d x \leq \frac{1}{\lambda} \int_{\Omega} f(x) d x .
$$

This shows that

$$
\left.|\nabla u|^{p} / u^{m} \in L^{1}(\Omega) \quad \text { (i.e. }\left|\nabla u^{(p-m) / p}\right| \in L^{p}(\Omega)\right) .
$$

The following lemma is the key to the whole proof.

Lemma 3.6. $\nabla U_{\epsilon}^{(p-m) / p} \rightarrow \nabla u^{(p-m) / p}$ strongly in $L^{p}(\Omega)\left(\epsilon \rightarrow 0^{+}\right)$.

To show Lemma 3.6, we need the following

Lemma 3.7. Let $\Phi$ be as in Lemma 3.2. Then

$$
\int_{\Omega}[\Phi(x)]^{-s} d x<\infty \quad \text { if and only if } \quad s<1 .
$$

Proof. We first claim that

$$
\int_{\Omega}[d(x)]^{-s} d x<\infty \text { if and only if } s<1,
$$

where $d(x)=\operatorname{dist}(x, \partial \Omega)$. Denote by $\Psi_{1}$ the eigenfunction corresponding to the first eigenvalue $\lambda_{1}$ of $-\Delta$ in $\Omega$ with homogeneous Dirichlet boundary condition. Theorem 2 from $[\mathrm{V}]$ yields $\partial \Psi_{1} / \partial \nu>0$ on $\partial \Omega$, where $\nu$ denotes the interior unit normal to $\partial \Omega$, so $\Psi_{1}(x) \geq C d(x)$ for all $x \in \bar{\Omega}$. On the other hand, since $\Psi_{1} \in C^{1}(\bar{\Omega})$, we have $\Psi_{1}(x) \leq C\left|x-x_{0}\right|$ for any $x_{0} \in \partial \Omega$ and for all $x \in \bar{\Omega}$. In addition, there exists some $\bar{x} \in \partial \Omega$ such that $d(x)=|x-\bar{x}|$, 
and hence $\Psi_{1}(x) \leq C d(x)$ for all $x \in \bar{\Omega}$. Since (see [LM])

$$
\int_{\Omega}\left[\Psi_{1}(x)\right]^{-s} d x<\infty \text { if and only if } s<1,
$$

we find that $(3.15)$ holds.

Theorem 5 from [V] yields $\partial \Phi / \partial \nu>0$ on $\partial \Omega$, hence $\Phi(x) \geq C d(x)$ for all $x \in \bar{\Omega}$. By the same reasoning as for $\Psi_{1}$, one can derive that $\Phi(x) \leq C d(x)$ for all $x \in \bar{\Omega}$. Therefore, there exist two positive constants $C_{2}, C_{1}$ with $C_{2} \geq C_{1}$ such that

$$
C_{1} d(x) \leq \Phi(x) \leq C_{2} d(x), \quad \forall x \in \bar{\Omega} .
$$

This and 3.15) imply the desired result.

Proof of Lemma 3.6. Clearly, we have

$$
\nabla U_{\epsilon}^{(p-m) / p} \rightarrow \nabla u^{(p-m) / p} \quad \text { weakly in } L^{p}(\Omega)\left(\epsilon \rightarrow 0^{+}\right) .
$$

Let $V_{\epsilon}=u+\epsilon$. Note that for fixed $\epsilon \in(0,1 / 2)$,

$$
\varphi=\left(\frac{p-m}{p}\right)^{p-1} U_{\epsilon}^{-(p-1) m / p}\left(U_{\epsilon}^{(p-m) / p}-V_{\epsilon}^{(p-m) / p}\right) \in \mathcal{F}_{0} .
$$

Substituting it into 3.3 yields

$$
\begin{aligned}
& \int_{\Omega}\left|\nabla U_{\epsilon}^{(p-m) / p}\right|^{p-2} \nabla U_{\epsilon}^{(p-m) / p} \nabla\left(U_{\epsilon}^{(p-m) / p}-V_{\epsilon}^{(p-m) / p}\right) d x \\
= & \left(\frac{p-m}{p}\right)^{p-1} \frac{(p-1) m}{p} \int_{\Omega} \frac{\left|\nabla U_{\epsilon}\right|^{p}}{U_{\epsilon}^{(p+(p-1) m) / p}}\left(U_{\epsilon}^{(p-m) / p}-V_{\epsilon}^{(p-m) / p}\right) d x \\
& -\lambda\left(\frac{p-m}{p}\right)^{p-1} \int_{\Omega} \frac{\left|\nabla U_{\epsilon}\right|^{p}}{U_{\epsilon}^{(2 p-1) m / p}}\left(U_{\epsilon}^{(p-m) / p}-V_{\epsilon}^{(p-m) / p}\right) d x \\
& +\left(\frac{p-m}{p}\right)^{p-1} \int_{\Omega} f(x) U_{\epsilon}^{-(p-1) m / p}\left(U_{\epsilon}^{(p-m) / p}-V_{\epsilon}^{(p-m) / p}\right) d x \\
:= & I_{1 \epsilon}+I_{2 \epsilon}+I_{3 \epsilon} .
\end{aligned}
$$

Below we shall show that

$$
\varlimsup_{\epsilon \rightarrow 0^{+}} \int_{\Omega}\left|\nabla U_{\epsilon}^{(p-m) / p}\right|^{p-2} \nabla U_{\epsilon}^{(p-m) / p} \nabla\left(U_{\epsilon}^{(p-m) / p}-V_{\epsilon}^{(p-m) / p}\right) d x \leq 0 .
$$

By the first estimate in (3.5), there exists a constant $\delta \in(0,1)$ such that for all $\epsilon \in(0, \tau)$, where $\tau=\min \left\{\frac{1}{2}, \frac{1}{2}\left(\frac{p \lambda}{(p-1) m}\right)^{1 /(m-1)}\right\}$, we have

$$
u_{\epsilon}<\tau \quad \text { in } \Omega_{\delta}:=\{x \in \Omega ; \operatorname{dist}(x, \partial \Omega)<\delta\},
$$

thus, for all $\epsilon \in(0, \tau)$,

$$
U_{\epsilon}^{m-1}=\left(u_{\epsilon}+\epsilon\right)^{m-1}<(2 \tau)^{m-1} \leq \frac{p \lambda}{(p-1) m} \quad \text { in } \Omega_{\delta} .
$$


This and $U_{\epsilon} \geq V_{\epsilon}$ imply that

$$
\begin{aligned}
\int_{\Omega_{\delta}} \frac{\left|\nabla U_{\epsilon}\right|^{p}}{U_{\epsilon}^{(p+(p-1) m) / p}} & \left(U_{\epsilon}^{(p-m) / p}-V_{\epsilon}^{(p-m) / p}\right) d x \\
& =\int_{\Omega_{\delta}} \frac{\left|\nabla U_{\epsilon}\right|^{p}}{U_{\epsilon}^{(2 p-1) m / p}} U_{\epsilon}^{m-1}\left(U_{\epsilon}^{(p-m) / p}-V_{\epsilon}^{(p-m) / p}\right) d x \\
& \leq \frac{p \lambda}{(p-1) m} \int_{\Omega_{\delta}} \frac{\left|\nabla U_{\epsilon}\right|^{p}}{U_{\epsilon}^{(2 p-1) m / p}}\left(U_{\epsilon}^{(p-m) / p}-V_{\epsilon}^{(p-m) / p}\right) d x
\end{aligned}
$$

for all $\epsilon \in(0, \tau)$, therefore

$$
\begin{aligned}
I_{1 \epsilon}= & \left(\frac{p-m}{p}\right)^{p-1} \frac{(p-1) m}{p} \int_{\Omega_{\delta}} \frac{\left|\nabla U_{\epsilon}\right|^{p}}{U_{\epsilon}^{(p+(p-1) m) / p}}\left(U_{\epsilon}^{(p-m) / p}-V_{\epsilon}^{(p-m) / p}\right) d x \\
& +\left(\frac{p-m}{p}\right)^{p-1} \frac{(p-1) m}{p} \int_{\Omega-\Omega_{\delta}} \frac{\left|\nabla U_{\epsilon}\right|^{p}}{U_{\epsilon}^{(p+(p-1) m) / p}}\left(U_{\epsilon}^{(p-m) / p}-V_{\epsilon}^{(p-m) / p}\right) d x \\
\leq & \lambda\left(\frac{p-m}{p}\right)^{p-1} \int_{\Omega_{\delta}} \frac{\left|\nabla U_{\epsilon}\right|^{p}}{U_{\epsilon}^{(2 p-1) m / p}}\left(U_{\epsilon}^{(p-m) / p}-V_{\epsilon}^{(p-m) / p}\right) d x \\
& +\left(\frac{p-m}{p}\right)^{p-1} \frac{(p-1) m}{p} \int_{\Omega-\Omega_{\delta}} \frac{\left|\nabla U_{\epsilon}\right|^{p}}{U_{\epsilon}^{(p+(p-1) m) / p}}\left(U_{\epsilon}^{(p-m) / p}-V_{\epsilon}^{(p-m) / p}\right) d x \\
\leq & -I_{2 \epsilon}+\left(\frac{p-m}{p}\right)^{p-1} \frac{(p-1) m}{p} \int_{\Omega-\Omega_{\delta}} \frac{\left|\nabla U_{\epsilon}\right|^{p}}{U_{\epsilon}^{(p+(p-1) m) / p}}\left(U_{\epsilon}^{(p-m) / p}-V_{\epsilon}^{(p-m) / p}\right) d x,
\end{aligned}
$$

i.e.

(3.18) $\quad I_{1 \epsilon}+I_{2 \epsilon}$

$$
\leq\left(\frac{p-m}{p}\right)^{p-1} \frac{(p-1) m}{p} \int_{\Omega-\Omega_{\delta}} \frac{\left|\nabla U_{\epsilon}\right|^{p}}{U_{\epsilon}^{(p+(p-1) m) / p}}\left(U_{\epsilon}^{(p-m) / p}-V_{\epsilon}^{(p-m) / p}\right) d x .
$$

On the other hand, by (3.13) and (3.6), we have

$$
\begin{aligned}
\int_{\Omega-\Omega_{\delta}} \frac{\left|\nabla U_{\epsilon}\right|^{p}}{U_{\epsilon}^{(p+(p-1) m) / p}} & \left(U_{\epsilon}^{(p-m) / p}-V_{\epsilon}^{(p-m) / p}\right) d x \\
& =\int_{\Omega-\Omega_{\delta}} \frac{\left|\nabla U_{\epsilon}\right|^{p}}{U_{\epsilon}^{m}}\left[1-\left(\frac{V_{\epsilon}}{U_{\epsilon}}\right)^{(p-m) / p}\right] d x \\
& \leq C \frac{\max }{\Omega-\Omega_{\delta}}\left[1-\left(\frac{V_{\epsilon}}{U_{\epsilon}}\right)^{(p-m) / p}\right] \rightarrow 0 \quad\left(\epsilon \rightarrow 0^{+}\right) .
\end{aligned}
$$


This together with 3.18$)$ implies that

$$
\varlimsup_{\epsilon \rightarrow 0^{+}}\left(I_{1 \epsilon}+I_{2 \epsilon}\right) \leq 0 .
$$

Next let us estimate $I_{3 \epsilon}$. Since $1<m<2 p /(p+1)$, it follows that $p(m-1) /(p-m)<1$. By Lemma 3.7, one obtains

$$
\left.\int_{\Omega} \Phi^{p(1-m) /(p-m)} d x<\infty \quad \text { (i.e. } \Phi^{p(1-m) /(p-m)} \in L^{1}(\Omega)\right) .
$$

Since $U_{\epsilon} \geq V_{\epsilon} \geq \epsilon$, by noticing $m>1$ and by using the second estimate in (3.5), we have

$$
0 \leq U_{\epsilon}^{-(p-1) m / p}\left(U_{\epsilon}^{(p-m) / p}-V_{\epsilon}^{(p-m) / p}\right) \leq U_{\epsilon}^{1-m} \leq C \Phi^{p(1-m) /(p-m)} \text { in } \Omega .
$$

Note that

$$
U_{\epsilon}^{-(p-1) m / p}\left(U_{\epsilon}^{(p-m) / p}-V_{\epsilon}^{(p-m) / p}\right) \rightarrow 0 \quad \text { in } \Omega\left(\epsilon \rightarrow 0^{+}\right) .
$$

Using Lebesgue's dominated convergence theorem, we get

$$
I_{3 \epsilon}=\int_{\Omega} f(x) U_{\epsilon}^{-(p-1) m / p}\left(U_{\epsilon}^{(p-m) / p}-V_{\epsilon}^{(p-m) / p}\right) d x \rightarrow 0 \quad\left(\epsilon \rightarrow 0^{+}\right) .
$$

This together with (3.16) and (3.19) implies (3.17).

Noticing

$$
0 \leq 1-\left(\frac{u}{V_{\epsilon}}\right)^{m / p} \leq 1 \quad \text { in } \Omega, \quad 1-\left(\frac{u}{V_{\epsilon}}\right)^{m / p} \rightarrow 0 \quad \text { in } \Omega \quad\left(\epsilon \rightarrow 0^{+}\right),
$$

and using Lebesgue's dominated convergence theorem and (3.14), we obtain, as $\epsilon \rightarrow 0^{+}$,

$$
\int_{\Omega}\left|\nabla\left(u^{(p-m) / p}-V_{\epsilon}^{(p-m) / p}\right)\right|^{p} d x=\left(\frac{p-m}{p}\right)^{p} \int_{\Omega} \frac{|\nabla u|^{p}}{u^{m}}\left|1-\left(\frac{u}{V_{\epsilon}}\right)^{m / p}\right|^{p} d x \rightarrow 0,
$$

therefore, by Hölder's inequality and by using (3.13), we have

$$
\begin{aligned}
& \left.\left|\int_{\Omega}\right| \nabla U_{\epsilon}^{(p-m) / p}\right|^{p-2} \nabla U_{\epsilon}^{(p-m) / p} \nabla\left(u^{(p-m) / p}-V_{\epsilon}^{(p-m) / p}\right) d x \mid \\
& \leq\left(\int_{\Omega}\left|\nabla U_{\epsilon}^{(p-m) / p}\right|^{p} d x\right)^{(p-1) / p} \cdot\left(\int_{\Omega}\left|\nabla\left(u^{(p-m) / p}-V_{\epsilon}^{(p-m) / p}\right)\right|^{p} d x\right)^{1 / p} \\
& \leq C\left(\int_{\Omega}\left|\nabla\left(u^{(p-m) / p}-V_{\epsilon}^{(p-m) / p}\right)\right|^{p} d x\right)^{1 / p} \rightarrow 0 \quad\left(\epsilon \rightarrow 0^{+}\right) .
\end{aligned}
$$

From this and (3.17) it follows that 


$$
\varlimsup_{\epsilon \rightarrow 0} \int_{\Omega}\left|\nabla U_{\epsilon}^{(p-m) / p}\right|^{p-2} \nabla U_{\epsilon}^{(p-m) / p} \nabla\left(U_{\epsilon}^{(p-m) / p}-u^{(p-m) / p}\right) d x \leq 0 .
$$

Since

$$
\lim _{\epsilon \rightarrow 0^{+}} \int_{\Omega}\left|\nabla u^{(p-m) / p}\right|^{p-2} \nabla u^{(p-m) / p} \nabla\left(U_{\epsilon}^{(p-m) / p}-u^{(p-m) / p}\right) d x=0,
$$

we derive that

$$
\begin{aligned}
\varlimsup_{\epsilon \rightarrow 0^{+}} \int_{\Omega}\left(\left|\nabla U_{\epsilon}^{(p-m) / p}\right|^{p-2} \nabla U_{\epsilon}^{(p-m) / p}\right. & \left.-\left|\nabla u^{(p-m) / p}\right|^{p-2} \nabla u^{(p-m) / p}\right) \\
& \times \nabla\left(U_{\epsilon}^{(p-m) / p}-u^{(p-m) / p}\right) d x \leq 0 .
\end{aligned}
$$

Recalling the inequality 2.1, we have

$$
\begin{aligned}
\lim _{\epsilon \rightarrow 0^{+}} \int_{\Omega}\left(\left|\nabla U_{\epsilon}^{(p-m) / p}\right|^{p-2} \nabla U_{\epsilon}^{(p-m) / p}\right. & \left.-\left|\nabla u^{(p-m) / p}\right|^{p-2} \nabla u^{(p-m) / p}\right) \\
& \times \nabla\left(U_{\epsilon}^{(p-m) / p}-u^{(p-m) / p}\right) d x=0 .
\end{aligned}
$$

Then the same reasoning as in Lemma 3.4 completes the proof.

Lemma 3.6 immediately implies that

$$
\int_{\Omega} \frac{\left|\nabla u_{\epsilon}\right|^{p}}{\left(u_{\epsilon}+\epsilon\right)^{m}} d x \rightarrow \int_{\Omega} \frac{|\nabla u|^{p}}{u^{m}} d x \quad\left(\epsilon \rightarrow 0^{+}\right) .
$$

Then it is easy to check that $u$ is a solution of problem (1.1).

It remains to show that $u$ is maximal. Let $w$ be a solution of problem (1.1). Then $w_{\epsilon}=w+\epsilon$ satisfies

$$
\int_{\Omega}\left(\left|\nabla w_{\epsilon}\right|^{p-2} \nabla w_{\epsilon} \nabla \varphi+\frac{\lambda}{w_{\epsilon}^{m}}\left|\nabla w_{\epsilon}\right|^{p} \varphi-f(x) \varphi\right) d x \leq 0, \quad \forall \varphi \in \mathcal{F}_{0}^{+} .
$$

Recalling Proposition 2.4 and $(3.3)$, we see that

$$
U_{\epsilon}=u_{\epsilon}+\epsilon \geq w_{\epsilon}=w+\epsilon \quad \text { a.e. in } \Omega \text {, }
$$

i.e. $u_{\epsilon} \geq w$ a.e. in $\Omega$ and passing to the limit in $\epsilon$ gives $u \geq w$ a.e. in $\Omega$. The proof of Theorem 2.6 is complete.

4. Proof of Theorem 2.7. Under the hypotheses of Theorem 2.7, we first show the existence of a positive solution for problem (1.2) and 1.3 . Let $\epsilon \in(0,1)$, and define $\bar{H}_{\epsilon}:(0,1) \times \mathbb{R} \times \mathbb{R} \rightarrow \mathbb{R}$ by

$$
\bar{H}_{\epsilon}(r, v, \xi)=-\frac{N-1}{r+\epsilon^{1 / \alpha}}|\xi|^{p-2} \xi+\lambda \frac{|\xi|^{p}}{\left[I_{\epsilon}(v)\right]^{m}}-\bar{f}(r),
$$


where $\alpha=p /(p-m)$, and $I_{\epsilon}(v)=v+\epsilon^{2}$ if $v \geq 0, I_{\epsilon}(v)=\epsilon^{2}$ if $v<0$. By the inequality $a^{p-1} \leq a^{p}+1(a \geq 0)$, we have

$$
\begin{aligned}
\left|\bar{H}_{\epsilon}(r, v, \xi)\right| & \leq \frac{N-1}{\epsilon^{1 / \alpha}}|\xi|^{p-1}+\frac{\lambda}{\epsilon^{2 m}}|\xi|^{p}+\max _{[0,1]} \bar{f} \\
& \leq \frac{N-1}{\epsilon^{1 / \alpha}}\left(1+|\xi|^{p}\right)+\frac{\lambda}{\epsilon^{2 m}}|\xi|^{p}+\max _{[0,1]} \bar{f} \\
& \leq\left(\frac{N-1}{\epsilon^{1 / \alpha}}+\frac{\lambda}{\epsilon^{2 m}}+\max _{[0,1]} \bar{f}\right) \mathcal{H}(|\xi|)
\end{aligned}
$$

for all $(r, v, \xi) \in(0,1) \times \mathbb{R} \times \mathbb{R}$, where $\mathcal{H}(s)=1+s^{p}$ for $s \geq 0$. Denote $\mathcal{M}=\left\{u \in C^{1}(0,1) ;\left|u^{\prime}\right|^{p-2} u^{\prime} \in C^{1}(0,1)\right\}$ and define an operator $\mathcal{L}_{\epsilon}: \mathcal{M} \rightarrow$ $C(0,1)$ by

$$
\left(\mathcal{L}_{\epsilon} u\right)(r)=-\left(\left|u^{\prime}\right|^{p-2} u^{\prime}\right)^{\prime}+\bar{H}_{\epsilon}\left(r, u, u^{\prime}\right), \quad 0<r<1 .
$$

Consider the problem

$$
\left\{\begin{array}{l}
\left(\mathcal{L}_{\epsilon} u\right)(r)=0, \quad 0<r<1, \\
u(1)=u(0)=0 .
\end{array}\right.
$$

A function $u$ is called a sup-solution [sub-solution] of problem $(4.2)$ if $\mathcal{L}_{\epsilon} u \geq$ $[\leq] 0$ in $(0,1)$, and $u(r) \geq[\leq] 0$ at $r=0,1$.

We will apply the sub-sup solutions method (cf. Theorem 1 and Remark 2.4 in [JG] to show the existence of solutions of problem (4.2). Since $\int_{0}^{\infty}\left(s^{p-1} / \mathcal{H}(r)\right) d r=\infty$, the condition (2.3) in [JG] is satisfied. Then it suffices to find a sub-solution and a sup-solution to obtain a solution.

Lemma 4.1. Let $W=C \Psi^{\alpha}$ with $\alpha=p /(p-m)$, where

$$
\Psi(r)=\frac{p-1}{p}\left[\left(\frac{1}{2}\right)^{p /(p-1)}-\left|\frac{1}{2}-r\right|^{p /(p-1)}\right],
$$

and the constant $C \in(0,1)$ is such that

$$
(C \alpha)^{p-1}+(N-1)(C \alpha)^{p-1} 2^{(\alpha-1)(p-1)-1}+\lambda C^{p-m} \alpha^{p} \leq \min _{[0,1]} \bar{f} .
$$

Then $W$ is a sub-solution of problem 4.2.

Proof. It is easy to check that $\Psi$ has the following properties:

(i) $\Psi>0$ in $(0,1), \Psi \in C^{1}[0,1]$,

(ii) $\left(\left|\Psi^{\prime}\right|^{p-2} \Psi^{\prime}\right)^{\prime}=-1$ in $(0,1), \Psi(1)=\Psi(0)=0$,

(iii) $|\Psi(r)| \leq r$ and $\left|\Psi^{\prime}(r)\right| \leq 1$, for all $r \in[0,1]$. 
Using the properties of $\Psi$ and noticing $(\alpha-1)(p-1)>1$, one arrives at

$$
\begin{aligned}
\mathcal{L}_{\epsilon} W= & -\left(\left|W^{\prime}\right|^{p-2} W^{\prime}\right)^{\prime}-\frac{N-1}{r+\epsilon^{1 / \alpha}}\left|W^{\prime}\right|^{p-2} W^{\prime}+\frac{\lambda}{\left(W+\epsilon^{2}\right)^{m}}\left|W^{\prime}\right|^{p}-\bar{f}(r) \\
\leq & -\left(\left|W^{\prime}\right|^{p-2} W^{\prime}\right)^{\prime}-\frac{N-1}{r+\epsilon^{1 / \alpha}}\left|W^{\prime}\right|^{p-2} W^{\prime}+\frac{\lambda}{W^{m}}\left|W^{\prime}\right|^{p}-\bar{f}(r) \\
= & -(C \alpha)^{p-1} \Psi^{(\alpha-1)(p-1)}\left(\left|\Psi^{\prime}\right|^{p-2} \Psi^{\prime}\right)^{\prime} \\
& -(C \alpha)^{p-1}(\alpha-1)(p-1) \Psi^{(\alpha-1)(p-1)-1}\left|\Psi^{\prime}\right|^{p} \\
& -(N-1)(C \alpha)^{p-1} \frac{\Psi^{(\alpha-1)(p-1)}}{r+\epsilon^{1 / \alpha}}\left|\Psi^{\prime}\right|^{p-2} \Psi^{\prime}+\lambda C^{p-m} \alpha^{p}\left|\Psi^{\prime}\right|^{p}-\bar{f}(r) \\
= & (C \alpha)^{p-1} \Psi^{(\alpha-1)(p-1)}-(C \alpha)^{p-1}(\alpha-1)(p-1) \Psi^{(\alpha-1)(p-1)-1}\left|\Psi^{\prime}\right|^{p} \\
& -(N-1)(C \alpha)^{p-1} \frac{\Psi^{(\alpha-1)(p-1)}}{r+\epsilon^{1 / \alpha}}\left|\Psi^{\prime}\right|^{p-2} \Psi^{\prime}+\lambda C^{p-m} \alpha^{p}\left|\Psi^{\prime}\right|^{p}-\bar{f}(r) \\
\leq & (C \alpha)^{p-1} \Psi^{(\alpha-1)(p-1)}+(N-1)(C \alpha)^{p-1}\left(r+\epsilon^{1 / \alpha}\right)^{(\alpha-1)(p-1)-1}\left|\Psi^{\prime}\right|^{p-1} \\
& +\lambda C^{p-m} \alpha^{p}\left|\Psi^{\prime}\right|^{p}-\min _{[0,1]} \bar{f}(r) \quad\left(\operatorname{note} \Psi(r) \leq r+\epsilon^{1 / \alpha}\right) \\
\leq & (C \alpha)^{p-1}+(N-1)(C \alpha)^{p-1} 2^{(\alpha-1)(p-1)-1}+\lambda C^{p-m} \alpha^{p}-\min _{[0,1]} \bar{f}(r) \\
\leq & 0, \quad 0<r<1 . \quad \quad 0 .
\end{aligned}
$$

Thus the lemma follows.

Let $\inf _{r \geq 1} \mathcal{X}(r) \equiv \delta$. Then it follows from the definition of infimum and $\lambda>\delta$ that for $\delta_{0}=(\lambda-\delta) / 2>0$, there exists some $C_{*} \geq 1$, such that

$$
\mathcal{X}\left(C_{*}\right)<\delta+\delta_{0}<\lambda .
$$

Lemma 4.2. There exists a constant $\epsilon_{0} \in(0,1)$ such that for any $\epsilon \in$ $\left(0, \epsilon_{0}\right), V_{\epsilon}=C_{*}\left(r+\epsilon^{1 / \alpha}\right)^{\alpha}$ is a sup-solution of problem 4.2).

Proof. Noticing $V_{\epsilon} \geq \epsilon$ and $(\alpha-1) p=\alpha m$, one has

$$
\begin{aligned}
\mathcal{L}_{\epsilon} V_{\epsilon}= & -\left(\left|V_{\epsilon}^{\prime}\right|^{p-2} V_{\epsilon}^{\prime}\right)^{\prime}-\frac{N-1}{r+\epsilon^{1 / \alpha}}\left|V_{\epsilon}^{\prime}\right|^{p-2} V_{\epsilon}^{\prime}+\frac{\lambda}{\left(V_{\epsilon}+\epsilon^{2}\right)^{m}}\left|V_{\epsilon}^{\prime}\right|^{p}-\bar{f} \\
= & -\left(C_{*} \alpha\right)^{p-1}(\alpha-1)(p-1)\left(r+\epsilon^{1 / \alpha}\right)^{(\alpha-1)(p-1)-1} \\
& -(N-1)\left(C_{*} \alpha\right)^{p-1}\left(r+\epsilon^{1 / \alpha}\right)^{(\alpha-1)(p-1)-1} \\
& +\frac{\lambda C_{*}^{p-m} \alpha^{p}}{\left[1+C_{*}^{-1}\left(r+\epsilon^{1 / \alpha}\right)^{-\alpha} \epsilon^{2}\right]^{m}}-\bar{f} .
\end{aligned}
$$


Since $(\alpha-1)(p-1)>1$, one obtains

$$
\begin{aligned}
\mathcal{L}_{\epsilon} V_{\epsilon} \geq & -\left(C_{*} \alpha\right)^{p-1}(\alpha-1)(p-1)\left(1+\epsilon^{1 / \alpha}\right)^{(\alpha-1)(p-1)-1} \\
& -(N-1)\left(C_{*} \alpha\right)^{p-1}\left(1+\epsilon^{1 / \alpha}\right)^{(\alpha-1)(p-1)-1}+\frac{\lambda C_{*}^{p-m} \alpha^{p}}{\left(1+C_{*}^{-1} \epsilon\right)^{m}}-\max _{[0,1]} \bar{f} \\
= & \lambda C_{*}^{p-m} \alpha^{p}-\left(C_{*} \alpha\right)^{p-1}[(\alpha-1)(p-1)+N-1]-\max _{[0,1]} \bar{f}+r(\epsilon) \\
= & C_{*}^{p-m} \alpha^{p}\left[\lambda-\mathcal{X}\left(C_{*}\right)\right]+r(\epsilon), \quad 0<r<1,
\end{aligned}
$$

where

$$
\begin{aligned}
r(\epsilon)= & \left(C_{*} \alpha\right)^{p-1}[(\alpha-1)(p-1)+N-1]\left[1-\left(1+\epsilon^{1 / \alpha}\right)^{(\alpha-1)(p-1)-1}\right] \\
& +\lambda \alpha^{p} C_{*}^{p-m}\left[\left(1+C_{*}^{-1} \epsilon\right)^{-m}-1\right] .
\end{aligned}
$$

Clearly, $r(\epsilon) \rightarrow 0$ as $\epsilon \rightarrow 0^{+}$. Since $\lambda>\mathcal{X}\left(C_{*}\right)$, there exists a constant $\epsilon_{0} \in(0,1)$ such that for any $\epsilon \in\left(0, \epsilon_{0}\right)$,

$$
C_{*}{ }^{p-m} \alpha^{p}\left[\lambda-\mathcal{X}\left(C_{*}\right)\right]+r(\epsilon) \geq 0, \quad 0<r<1 .
$$

Therefore for any $\epsilon \in\left(0, \epsilon_{0}\right)$,

$$
\mathcal{L}_{\epsilon} V_{\epsilon} \geq 0, \quad 0<r<1 .
$$

This shows that for any $\epsilon \in\left(0, \epsilon_{0}\right), V_{\epsilon}$ is a sup-solution of 4.2$)$.

By Theorem 1 and Remark 2.4 in [JG] and Lemmas 4.1-4.2, we see that for any fixed $\epsilon \in\left(0, \epsilon_{0}\right)$, problem 4.2 has a solution $u_{\epsilon} \in C^{1}[0,1] \cap \mathcal{M}$ with

$$
C_{*}\left(r+\epsilon^{1 / \alpha}\right)^{\alpha} \geq u_{\epsilon} \geq C \Psi^{\alpha}, \quad r \in[0,1] .
$$

Hence $u_{\epsilon}$ satisfies

$$
-\left(\left|u_{\epsilon}^{\prime}\right|^{p-2} u_{\epsilon}^{\prime}\right)^{\prime}-(N-1) \frac{\left|u_{\epsilon}^{\prime}\right|^{p-2} u_{\epsilon}^{\prime}}{r+\epsilon^{1 / \alpha}}+\lambda \frac{\left|u_{\epsilon}^{\prime}\right|^{p}}{\left(u_{\epsilon}+\epsilon^{2}\right)^{m}}=\bar{f}(r), r \in(0,1) .
$$

Next we estimate $u_{\epsilon}^{\prime}$. We first obtain

Lemma 4.3. There exists a positive constant $C$, independent of $\epsilon$, such that for all $\epsilon \in\left(0, \epsilon_{0}\right)$,

$$
\left|u_{\epsilon}^{\prime}(r)\right| \leq C, \quad \forall r \in[0,1] .
$$

Proof. Noticing $u_{\epsilon}(1)=u_{\epsilon}(0)=0$ and $u_{\epsilon} \geq 0$ on $[0,1]$, we have

$$
u_{\epsilon}^{\prime}(0) \geq 0 \geq u_{\epsilon}^{\prime}(1) \text {. }
$$

Integrating (4.5) over $(0,1)$ yields

$$
-\left.\left(\left|u_{\epsilon}^{\prime}\right|^{p-2} u_{\epsilon}^{\prime}\right)\right|_{0} ^{1}+\lambda \int_{0}^{1} \frac{\left|u_{\epsilon}^{\prime}\right|^{p}}{\left(u_{\epsilon}+\epsilon^{2}\right)^{m}} d r=(N-1) \int_{0}^{1} \frac{\left|u_{\epsilon}^{\prime}\right|^{p-2} u_{\epsilon}^{\prime}}{r+\epsilon^{1 / \alpha}} d r+\int_{0}^{1} \bar{f}(r) d r,
$$


and by (4.7), we derive that

$$
\lambda \int_{0}^{1} \frac{\left|u_{\epsilon}^{\prime}\right|^{p}}{\left(u_{\epsilon}+\epsilon^{2}\right)^{m}} d r \leq(N-1) \int_{0}^{1} \frac{\left|u_{\epsilon}^{\prime}\right|^{p-1}}{r+\epsilon^{1 / \alpha}} d r+\int_{0}^{1} \bar{f}(r) d r .
$$

Using Young's inequality $a b \leq \sigma a^{l}+\sigma^{-q / l} b^{q}(a, b \geq 0, \sigma>0, q, l>1$, $1 / l+1 / q=1)$ and taking $l=p /(p-1)$ and $q=p$, we deduce that

$$
\frac{\left|u_{\epsilon}^{\prime}\right|^{p-1}}{r+\epsilon^{1 / \alpha}} \leq \sigma \frac{\left|u_{\epsilon}^{\prime}\right|^{p}}{\left(r+\epsilon^{1 / \alpha}\right)^{p /(p-1)}}+\sigma^{1-p} .
$$

On the other hand, by the first estimate in (4.4) we obtain

$$
u_{\epsilon}(r)+\epsilon^{2} \leq C_{*}\left(r+\epsilon^{1 / \alpha}\right)^{\alpha}+\epsilon^{2} \leq 2 C_{*}\left(r+\epsilon^{1 / \alpha}\right)^{\alpha}, \quad r \in[0,1],
$$

therefore, due to $(p-m) /(p-1)<m$, we get

$$
\frac{\left|u_{\epsilon}^{\prime}\right|^{p}}{\left(r+\epsilon^{1 / \alpha}\right)^{p /(p-1)}} \leq C \frac{\left|u_{\epsilon}^{\prime}\right|^{p}}{\left(u_{\epsilon}+\epsilon^{2}\right)^{(p-m) /(p-1)}} \leq C \frac{\left|u_{\epsilon}^{\prime}\right|^{p}}{\left(u_{\epsilon}+\epsilon^{2}\right)^{m}}, \quad r \in[0,1],
$$

where $C>0$ are constants independent of $\epsilon$. Combining this and 4.9 yields

$$
\frac{\left|u_{\epsilon}^{\prime}\right|^{p-1}}{r+\epsilon^{1 / \alpha}} \leq \sigma C \frac{\left|u_{\epsilon}^{\prime}\right|^{p}}{\left(u_{\epsilon}+\epsilon^{2}\right)^{m}}+\sigma^{1-p}, \quad r \in[0,1] .
$$

Now taking $\sigma=\frac{\lambda}{2(N-1) C}$ in 4.11, we deduce from 4.8 that

$$
\frac{\lambda}{2} \int_{0}^{1} \frac{\left|u_{\epsilon}^{\prime}\right|^{p}}{\left(u_{\epsilon}+\epsilon^{2}\right)^{m}} d r \leq(N-1)\left(\frac{\lambda}{2(N-1) C}\right)^{1-p}+\int_{0}^{1} \bar{f}(r) d r \leq C .
$$

This and 4.11) imply that

$$
\int_{0}^{1} \frac{\left|u_{\epsilon}^{\prime}\right|^{p-1}}{r+\epsilon^{1 / \alpha}} d r \leq C
$$

Integrating (4.5) over $\left(r_{1}, r_{2}\right)$ and integrating by parts, we have

$$
\left.\left(\left|u_{\epsilon}^{\prime}\right|^{p-2} u_{\epsilon}^{\prime}\right)\right|_{r_{1}} ^{r_{2}}=\int_{r_{1}}^{r_{2}}\left(\frac{\lambda}{\left(u_{\epsilon}+\epsilon^{2}\right)^{m}}\left|u_{\epsilon}^{\prime}\right|^{p}-\frac{N-1}{r+\epsilon^{1 / \alpha}}\left|u_{\epsilon}^{\prime}\right|^{p-2} u_{\epsilon}^{\prime}-\bar{f}(r)\right) .
$$

Combining this with 4.12 and 4.13 , we find that there exists a positive constant $C$, independent of $\epsilon$, such that

$$
\left.|| u_{\epsilon}^{\prime}\left(r_{2}\right)\right|^{p-2} u_{\epsilon}^{\prime}\left(r_{2}\right)-\left|u_{\epsilon}^{\prime}\left(r_{1}\right)\right|^{p-2} u_{\epsilon}^{\prime}\left(r_{1}\right) \mid \leq C, \quad \forall r_{2}, r_{1} \in[0,1] .
$$

Since $u_{\epsilon}(0)=u_{\epsilon}(1)=0$ and $u_{\epsilon} \in C^{1}[0,1]$, by the mean value theorem there exists $r_{\epsilon} \in(0,1)$ such that $u_{\epsilon}^{\prime}\left(r_{\epsilon}\right)=0$. Then taking $r_{1}=r_{\epsilon}$ in (4.14) gives

$$
\left.|| u_{\epsilon}^{\prime}(r)\right|^{p-2} u_{\epsilon}^{\prime}(r) \mid \leq C, \quad \forall r \in[0,1] .
$$

This shows that $\left|u_{\epsilon}^{\prime}(r)\right| \leq C$ for all $r \in[0,1]$. 
Lemma 4.4. For any $\delta \in(0,1 / 2)$, there exists a positive constant $C_{\delta}$, independent of $\epsilon$, such that for all $\epsilon \in\left(0, \epsilon_{0}\right)$,

$$
\left|u_{\epsilon}^{\prime}\left(r_{2}\right)-u_{\epsilon}^{\prime}\left(r_{1}\right)\right| \leq C_{\delta}\left|r_{2}-r_{1}\right|^{\beta}, \quad \forall r_{2}, r_{1} \in[\delta, 1-\delta],
$$

where $\beta=1 /(p-1)$ if $p \geq 2, \beta=1$ if $1<p<2$.

Proof. By (4.4) and (4.6), one derives from (4.5) that for any $\delta \in(0,1 / 2)$, there exists a positive constant $C_{\delta}$, independent of $\epsilon$, such that for all $\epsilon \epsilon$ $\left(0, \epsilon_{0}\right)$,

$$
\left|\left(\left|u_{\epsilon}^{\prime}\right|^{p-2} u_{\epsilon}^{\prime}\right)^{\prime}\right| \leq C_{\delta}, \quad \delta \leq t \leq 1-\delta .
$$

Recalling the inequality (2.1) and using (4.16), one derives that if $p \geq 2$, then

$$
\begin{aligned}
\mid u_{\epsilon}^{\prime}\left(r_{2}\right) & -\left.u_{\epsilon}^{\prime}\left(r_{1}\right)\right|^{p} \\
& \leq C^{-1}\left[u_{\epsilon}^{\prime}\left(r_{2}\right)-u_{\epsilon}^{\prime}\left(r_{1}\right)\right] \cdot\left[\left|u_{\epsilon}^{\prime}\left(r_{2}\right)\right|^{p-2} u_{\epsilon}^{\prime}\left(r_{2}\right)-\left|u_{\epsilon}^{\prime}\left(r_{1}\right)\right|^{p-2} u_{\epsilon}^{\prime}\left(r_{1}\right)\right] \\
& \leq C_{\delta}\left|u_{\epsilon}^{\prime}\left(r_{2}\right)-u_{\epsilon}^{\prime}\left(r_{1}\right)\right|\left|r_{2}-r_{1}\right|, \quad \forall r_{2}, r_{1} \in[\delta, 1-\delta],
\end{aligned}
$$

so

$$
\left|u_{\epsilon}^{\prime}\left(r_{2}\right)-u_{\epsilon}^{\prime}\left(r_{1}\right)\right| \leq C_{\delta}\left|r_{2}-r_{1}\right|^{1 /(p-1)}, \quad \forall r_{2}, r_{1} \in[\delta, 1-\delta],
$$

and if $p \in(1,2)$, then

$$
\begin{aligned}
\mid u_{\epsilon}^{\prime}\left(r_{2}\right) & -\left.u_{\epsilon}^{\prime}\left(r_{1}\right)\right|^{2}\left[\left|u_{\epsilon}^{\prime}\left(r_{2}\right)\right|+\left|u_{\epsilon}^{\prime}\left(r_{1}\right)\right|\right]^{p-2} \\
& \leq C^{-1}\left[u_{\epsilon}^{\prime}\left(r_{2}\right)-u_{\epsilon}^{\prime}\left(r_{1}\right)\right] \cdot\left[\left|u_{\epsilon}^{\prime}\left(r_{2}\right)\right|^{p-2} u_{\epsilon}^{\prime}\left(r_{2}\right)-\left|u_{\epsilon}^{\prime}\left(r_{1}\right)\right|^{p-2} u_{\epsilon}^{\prime}\left(r_{1}\right)\right] \\
& \leq C_{\delta}\left|u_{\epsilon}^{\prime}\left(r_{2}\right)-u_{\epsilon}^{\prime}\left(r_{1}\right)\right|\left|r_{2}-r_{1}\right|, \quad \forall r_{2}, r_{1} \in[\delta, 1-\delta],
\end{aligned}
$$

therefore, using (4.6) yields

$$
\left|u_{\epsilon}^{\prime}\left(r_{2}\right)-u_{\epsilon}^{\prime}\left(r_{1}\right)\right| \leq C_{\delta}\left|r_{2}-r_{1}\right|\left[\left|u_{\epsilon}^{\prime}\left(r_{2}\right)\right|+\left|u_{\epsilon}^{\prime}\left(r_{1}\right)\right|\right]^{2-p} \leq C_{\delta}\left|r_{2}-r_{1}\right|
$$

for all $r_{2}, r_{1} \in[\delta, 1-\delta]$.

Using (4.6) and (4.15) and the Arzelà-Ascoli theorem, there exists a subsequence of $\left\{u_{\epsilon}\right\}$, still denoted by $\left\{u_{\epsilon}\right\}$, and a function $u \in C^{1}(0,1) \cap$ $C[0,1]$ such that, as $\epsilon \rightarrow 0^{+}$,

$$
\begin{array}{ll}
u_{\epsilon} \rightarrow u & \text { uniformly in } C[0,1], \\
u_{\epsilon} \rightarrow u & \text { uniformly in } C^{1}[\delta, 1-\delta],
\end{array}
$$

where $\delta \in(0,1 / 2)$, and hence it follows from $u_{\epsilon}(1)=u_{\epsilon}(0)=0$ and 4.4 ) that $u(1)=u(0)=0$, and

$$
C r^{p /(p-m)} \geq u(r) \geq C[\Psi(r)]^{p /(p-m)}, \quad r \in[0,1],
$$

therefore $u>0$ in $(0,1)$ and $u^{\prime}(0)=\lim _{r \rightarrow 0} u(r) / r=0$. Thus, (1.3) is satisfied. 
We now show that $u$ satisfies (1.2). Integrating (4.5) over $\left(r_{0}, r\right)(0<$ $r_{0}, r<1$ ) and integrating by parts gives

$$
\begin{aligned}
\left|u_{\epsilon}^{\prime}(r)\right|^{p-2} u_{\epsilon}^{\prime}(r)= & \int_{r_{0}}^{r}\left(\lambda \frac{\left|u_{\epsilon}^{\prime}\right|^{p}}{\left(u_{\epsilon}+\epsilon^{2}\right)^{m}}-\frac{N-1}{r+\epsilon^{1 / \alpha}}\left|u_{\epsilon}^{\prime}\right|^{p-2} u_{\epsilon}^{\prime}-\bar{f}(r)\right) d r \\
& +\left|u_{\epsilon}^{\prime}\left(r_{0}\right)\right|^{p-2} u_{\epsilon}^{\prime}\left(r_{0}\right) .
\end{aligned}
$$

Letting $\epsilon \rightarrow 0^{+}$and using Lebesgue's dominated convergence theorem yield

$$
\begin{aligned}
\left|u^{\prime}(r)\right|^{p-2} u^{\prime}(r)= & \int_{r_{0}}^{r}\left(\lambda \frac{\left|u^{\prime}\right|^{p}}{u^{m}}-\frac{N-1}{r}\left|u^{\prime}\right|^{p-2} u^{\prime}-\bar{f}(r)\right) d r \\
& +\left|u^{\prime}\left(r_{0}\right)\right|^{p-2} u^{\prime}\left(r_{0}\right) .
\end{aligned}
$$

This shows that $\left|u^{\prime}(r)\right|^{p-2} u^{\prime}(r) \in C^{1}(0,1)$, hence $(1.2)$ is satisfied.

Next we prove that $u \in C^{1}[0,1]$. Letting $\epsilon \rightarrow 0^{+}$in $(4.12)$ and (4.13) and using Fatou's lemma yield

$$
\int_{0}^{1} \frac{\left|u^{\prime}\right|^{p}}{u^{m}} d r \leq C, \quad \int_{0}^{1} \frac{\left|u^{\prime}\right|^{p-1}}{r} d r \leq C .
$$

So, $\left.\left|u^{\prime}\right|\right|^{p} / u^{m},\left|u^{\prime}\right|^{p-2} u^{\prime} / r \in L^{1}[0,1]$. By $(4.19)$, the function $\omega(r)=\left|u^{\prime}(r)\right|^{p-2} u^{\prime}(r)$ $=\phi_{p}\left(u^{\prime}(r)\right)$ is absolutely continuous on $[0,1]$. Since $u^{\prime}(r)=\phi_{q}(\omega(r))$, where $1 / p+1 / q=1$, we see that $u^{\prime} \in C[0,1]$. Thus $u$ is a positive solution of problem $(1.2)$ and $(1.3)$.

Proof of Theorem 2.7. Let $w(x)=u(r)$ with $r=|x|$. Clearly, $w(0)=0$. Some calculations give

$$
\begin{aligned}
& \nabla w=u^{\prime}(r) \frac{x}{|x|}, \quad|\nabla w|=\left|u^{\prime}(r)\right|, \quad \text { in } B_{1} \backslash\{0\}, \\
& \operatorname{div}\left(|\nabla w|^{p-2} \nabla w\right)=\left(\left|u^{\prime}\right|^{p-2} u^{\prime}\right)^{\prime}+\frac{N-1}{r}\left|u^{\prime}\right|^{p-2} u^{\prime} \quad \text { in } B_{1} \backslash\{0\} .
\end{aligned}
$$

Thus $w$ satisfies

$$
-\operatorname{div}\left(|\nabla w|^{p-2} \nabla w\right)+\frac{\lambda}{w^{m}}|\nabla w|^{p}-\bar{f}(|x|)=0 \quad \text { in } B_{1} \backslash\{0\} .
$$

Since $u \in C^{1}[0,1]$ and $u^{\prime}(0)=0,|\nabla w(0)|=0$ and $w \in C^{1}\left(\bar{B}_{1}\right)$.

By the first estimate in 4.20 , one obtains $|\nabla w|^{p} / w^{m} \in L^{1}\left(B_{1}\right)$.

In virtue of the above facts, it is not difficult to check that $w$ is a solution of problem (1).

It remains to show 2.2. By a simple calculation, we obtain

$$
\Psi^{\prime}(r)=\left(\frac{1}{2}-r\right)^{1 /(p-1)} \geq\left(\frac{1}{4}\right)^{1 /(p-1)}, \quad \forall 0 \leq r \leq \frac{1}{4} .
$$


Then

$$
\Psi(r) \geq\left(\frac{1}{4}\right)^{1 /(p-1)} r, \quad \forall 0 \leq r \leq \frac{1}{4} .
$$

This together with 4.18 implies 2.2.

The proof of Theorem 2.7 is complete.

Acknowledgements. The authors would like to thank the referee for his/her careful reading of the manuscript and helpful comments. This research was partly supported by the Department of Education of Liaoning Province (grant no. 2009A152) and NNSFC (grant no. 10901030).

\section{References}

[ADP] B. Abdellaoui, A. Dall'Aglio and I. Peral, Some remarks on elliptic problems with critical growth in the gradient, J. Differential Equations 222 (2006), 21-62.

[AAA] L. Aharouch, Y. Akdim and E. Azroul, Quasilinear degenerate elliptic unilateralproblems, Abstract Appl. Anal. 2005, no. 1, 11-31.

[A] H. Amann, Existence and multiplicity theorems for semi-linear elliptic boundary value problems, Math. Z. 150 (1976), 281-295.

[AB] D. Arcoya and L. Boccardo, Critical points for multiple integrals of the calculus of variations, Arch. Ration. Mech. Anal. 134 (1996), 249-274.

[AMA] D. Arcoya and P. J. Martínez-Aparicio, Quasilinear equations with natural growth, Rev. Mat. Iberoamer. 24 (2008), 597-616.

[AM] C. Astarita and G. Marrucci, Principles of Non-Newtonian Fluid Mechanics, McGraw-Hill, 1974.

[BBM] A. Bensoussan, L. Boccardo and F. Murat, On a nonlinear partial differential equation having natural growth terms and unbounded solution, Ann. Inst. H. Poincaré Anal. Non Linéaire 5 (1988), 347-364.

[BMP1] L. Boccardo, F. Murat and J.-P. Puel, Existence of bounded solutions for nonlinear elliptic unilateral problems, Ann. Mat. Pura Appl. 152 (1988), 183-196.

[BMP2] - - - - , $L^{\infty}$ estimate for some nonlinear elliptic partial differential equations and application to an existence result, SIAM J. Math. Anal. 23 (1992), 326-333.

[BO] L. Boccardo and L. Orsina, Existence and regularity of minima for integral functionals noncoercive in the energy space, Ann. Scuola Norm. Sup. Pisa Cl. Sci. 25 (1997), 95-130.

[BST] L. Boccardo, S. Segura de León and C. Trombetti, Bounded and unbounded solutions for a class of quasi-linear elliptic problems with a quadratic gradient term, J. Math. Pures Appl. 80 (2001), 919-940.

[CC] K. Cho and H. J. Choe, Nonlinear degenerate elliptic partial differential equations with critical growth conditions on the gradient, Proc. Amer. Math. Soc. 123 (1995), 3789-3796.

[D] L. Damascelli, Comparison theorems for some quasilinear degenerate elliptic operations and applications to symmetry and monotonicity results, Ann. Inst. H. Poincaré Anal. Non Linéaire 15 (1998), 493-516.

[DB] E. DiBenedetto, $C^{1, \alpha}$ local regularity of weak solutions of degenerate elliptic equations, Nonlinear Anal. 7 (1983), 827-850. 
[DGP] A. Dall'Aglio, D. Giachetti and J.-P. Puel, Nonlinear elliptic equations with natural growth in general domains, Ann. Mat. Pura Appl. 181 (2002), 407-426.

[DN] P. Drábek and F. Nicolosi, Existence of bounded solutions for some degenerate quasilinear elliptic equations, ibid. 165 (1993), 217-238.

[FPR] V. Ferone, M. R. Posteraro and J.-M. Rakotoson, $L^{\infty}$-estimates for nonlinear elliptic problems with p-growth in the gradient, J. Inequal. Appl. 3 (1999), 109125.

[G] N. Grenon, Existence and comparison results for quasilinear elliptic equations with critical growth in the gradient, J. Differential Equations 171 (2001), 1-23.

[GT] N. Grenon and C. Trombetti, Existence results for a class of nonlinear elliptic problems with p-growth in the gradient, Nonlinear Anal. 52 (2003), 931-942.

[JG] D. Q. Jiang and W. J. Gao, Singular boundary value problems for the onedimension p-Laplacian, J. Math. Anal. Appl. 270 (2002), 561-581.

[LU] O. A. Ladyzhenskaya and N. N. Ural'tseva, Linear and Quasilinear Elliptic Equations, Academic Press, New York, 1968.

[LM] A. C. Lazer and P. J. McKenna, On a singular nonlinear elliptic boundary value problem, Proc. Amer. Math. Soc. 111 (1991), 721-730.

[L] G. M. Lieberman, Boundary regularity for solutions of degenerate elliptic equations, Nonlinear Anal. 12 (1988), 1203-1219.

[MP] L. K. Martinson and K. B. Pavlov, The effect of magnetic plasticity in nonNewtonian fluid, Magnit. Gidrodinamika 3 (1969), 69-75 (in Russian).

[MRS] B. Michaux, J.-M. Rakotoson and J. Shen, On the existence and regularity of solutions of a quasilinear mixed equation of Leray-Lions type, Acta Appl. Math. 12 (1988), 1572-9036.

[OP] L. Orsina and J.-P. Puel, Positive solutions for a class of nonlinear elliptic problems involving quasilinear and semilinear terms, Comm. Partial Differential Equations 26 (2001), 1665-1689.

[P] A. Porretta, Nonlinear equations with natural growth terms and measure data, in: Proceedings of the 2002 Fez Conference on Partial Differential Equations, Electron. J. Differential Equations Conf. 9 (2002), 183-202.

[PS] A. Porretta and S. Segura de León, Nonlinear elliptic equations having a gradient term with natural growth, J. Math. Pures Appl. 85 (2006), 465-492.

[PV] G. Porru and A. Vitolo, Problems for elliptic singular equations with a quadratic gradient term, J. Math. Anal. Appl. 334 (2007), 467-486.

[R] J.-M. Rakotoson, Rérrangement relatif dans les équations elliptiques quasilinéaires avec un second membre distribution: Application à un théorème d'existence et de régularité, J. Differential Equations 66 (1987), 391-419.

[RT] J.-M. Rakotoson and R. Temam, Relative rearrangement in quasilinear variational inequalities, Indiana Univ. Math. J. 36 (1987), 757-810.

[T] P. Tolksdorf, Regularity for a more general class of quasilinear elliptic equations, J. Differential Equations 51 (1984), 126-150.

[Tr] C. Trombetti, Non-uniformly elliptic equations with natural growth in the gradient, Potential Anal. 18 (2003), 391-404.

[V] J. L. Vázquez, A strong maximum principle for some quasilinear elliptic equations, Appl. Math. Optim. 12 (1984), 191-202.

[YC] H. J. Yuan and M. T. Chen, Positive solutions for a class of p-Laplace problems involving quasi-linear and semi-linear terms, J. Math. Anal. Appl. 330 (2007), 1179-1193.

[Z1] W. S. Zhou, A singular nonlinear elliptic equation with natrual growth in the gradient, Math. Methods Appl. Sci. 31 (2008), 1704-1721. 
[Z2] W. S. Zhou, Existence and multiplicity of weak solutions for a singular semilinear elliptic equation, J. Math. Anal. Appl. 346 (2008), 107-119.

Wen-shu Zhou, Xiao-dan Wei

Department of Mathematics

Dalian Nationalities University

116600 Dalian, P.R. China

E-mail: weixiaodancat@126.com

Received 6.9.2009

and in final form 6.12.2009 